\title{
¿CAPACITACIÓN O PROFESIONALIZACIÓN DE LOS MAESTROS DE FÍSICA EN EJERCICIO? ${ }^{1}$
}

Germán Bautista R.*

\begin{abstract}
A reflection on sorne ideas that support strategies that has been carried out by educational institutions is put forward. It is remarked that teachers' conceptions on science and knowledge play an important role on the way teachers accomplish their every day tasks.
\end{abstract}

\section{RESUMEN}

Se analizan algunos elementos que subyacen a las orientaciones y estrategias que las diversas instituciones educativas suelen levar a cabo para capacitar a los maestros en ejercicio con el ánimo de incidir en el mejoramiento de la calidad de la educación. Se destacan como elementos importantes de esta problemática la concepción que se tenga acerca de las ciencias y la consecuente concepción sobre el quehacer del maestro que ésta genera.

\section{INTRODUCCIÓN}

Que el bienestar de los miembros de una sociedad se constituye en una de las razones de ser de los diferentes organismos del Estado, es un consenso compartido por la inmensa mayoría de los países contemporáneos. Bienestar que suele ser relacionado (en mayor o menor grado, según las diferentes concepciones que sobre sociedad se asuman) con la capacidad de los individuos para acceder a más y mejores servicios y bienes. Para posibilitar la producción de bienes y servicios se suele asignar un papel determinante al desarrollo científico y tecnológico. Se establece, entonces, una relación directa entre el desarrollo de una sociedad y su desarrollo científico y tecnológico. ${ }^{2}$

En Colombia, por ejemplo, El Departamento de Planeación Nacional (COMPES) al presentar el "Plan de Apertura Educativa 1991-1994" afirma: "La experiencia ha

\footnotetext{
1 Trabajo presentado en la $\vee$ Reunión Latinoamericana sobre Enseñanza de la Física, Porto Alegre (Gramado). Agosto 24 a 28 de 1992.

Profesor Asociado Universidad Pedagógica Nacional. Departamento de Física. e-mail: bautista@uni.pedagogica.edu.co

${ }_{2}^{2}$ Véase por ejemplo la introducción al Vol. 2, Tomo I de ESTRUCTURA CIENTífICA, DESARROLLO TECNOLÓGICO Y ESTRUCTURA SOCIAL, Publicado por: Misión de Ciencia y Tecnología, Ministerio de Educación Nacional (M.E.N), Departamento Nacional de Planeación (D.N.P), y FONADE. Santa Fe de Bogotá, D.C. 1990.
} 
demostrado que la política social con efectos más perdurables sobre la marcha social es aquella que acrecienta las oportunidades económicas para la gente,..."3.

También se ha venido constituyendo en consenso el reconocimiento del importante papel que la educación desempeña en el desarrollo de la sociedad, es así, como el COMPES en su evaluación de la educación en el país, enfatiza la necesidad de mejorar su calidad y le señala un "papel primordial" en el fortalecimiento de la estructura social.

La consecuente creciente demanda de desarrollo científico y tecnológico de nuestro país hace cada vez más urgente diseñar estrategias que garanticen una mejor enseñanza de las ciencias.

\section{LA CAPACITACION}

Por otro lado, el paulatino reconocimiento del decisivo papel que el maestro desempeña en los procesos educativos, ${ }^{4}$ ha llevado a emprender múltiples acciones y estrategias encaminadas al mejoramiento de los docentes en ejercicio ${ }^{5}$. La capacitación de maestros ha sido desde hace algunos años un centro muy importante de las acciones que buscan mejorar la calidad de la educación. Capacitación que se realiza bajo el supuesto de que el problema básico reside en la deficiencia de los conocimientos en pedagogía pero especialmente en la correspondiente disciplina, (Física, para nuestro caso), que poseen los maestros en ejercicio. Se suelen presentar los siguientes argumentos para justificar la necesidad de capacitación:

- La deficiente formación de los maestros, con gran énfasis en el escaso manejo de la disciplina.

- La desactualización que el maestro sufre con el transcurrir del tiempo: debido a los acelerados desarrollos de la disciplina, o, debido al proceso natural de olvido de contenidos cuando no se los usa.

- La necesidad de implementar innovaciones educativas que se derivan de los avances en la investigación educativa.

- La necesidad de desarrollar los cambios que las autoridades educativas, obedeciendo a planes y políticas de desarrollo, intentan implantar.

Lo anterior ha llevado a múltiples acciones y propuestas, tanto de organismos nacionales como internacionales, sin que hasta el momento se hayan obtenido resultados satisfactorios. Los escasos resultados suelen ser explicados aduciendo los siguientes factores:

3 PLAN DE APERTURA EDUCATIVA 1991-1994. Documento M.E.N., D.N.P., U.D.S., D.E.C., 2518. Departamento Nacional de Planeación. Santa Fe de Bogotá D.C., marzo 19 de 1991.

${ }^{4}$ M. M. Ayala. LA ENSEÑANZA DE LA FÍSICA PARA LA FORMACIÓN DE PROFESORES DE FÍSICA. Trabajo presentado en la $V$ Reunión Latinoamericana sobre Enseñanza de la Física, Porto Alegre (Gramado). Agosto 24 al 28 de 1992.

${ }^{5} \mathrm{P}$. Pinilla. CONTRIBUCIÓN DE LA EDUCACIÓN BÁSICA Y MEDIA VOCACIONAL AL DESARROLLO DE LA CIENCIA Y LA TECNOLOGÍA. Estructura Científica y Desarrollo Tecnológico y Entorno Social Vol.2 Tomo 1. Editado por Departamento Nacional de Planeación: Misión de Ciencia y Tecnología. 1990. 
- Bajo nivel económico o de ingresos de los maestros en ejercicio.

- Poca receptividad por parte de los maestros de las investigaciones educativas y de los avances de las disciplinas.

- Imagen social y auto imagen del maestro (el problema de la identidad del maestro).

Ante los pobres resultados de las acciones emprendidas desde esta perspectiva, frecuentemente se destaca una actitud refractaria o de desánimo de los maestros a las innovaciones 6 .

Es interesante notar que tanto las motivaciones generalmente aducidas para la capacitación como los aspectos que se suelen resaltar cuando se evalúan las acciones que consecuentemente se desarrollan, son concordantes con aquellas concepciones filosóficas y epistemológicas que consideran a las ciencias y los saberes como los simples productos de una actividad. Esta visión, muy difundida, de las ciencias y la correspondiente concepción que ella genera sobre el rol de las ciencias en el entramado cultural de una sociedad particular conduce a asignar un peculiar papel al quehacer del maestro: el maestro como simple transmisor de saberes, de los cuales no puede dar cuenta.

\section{EL PROTAGONISMO DEL MAESTRO}

Se coloca, así, al maestro en un papel de impotencia, de no poder adueñar-se de esos saberes. Su problema y su radio de acción se reducen a transmitir no ha producir. La parte activa de la labor del maestro se reduce a la simple elaboración o aplicación de recursos metodológicos.

Por otro lado, refiriéndose a la participación del personal docente en la planeación académica y en el desarrollo escolar, P. Pínula anota: “... en contra del discurso que contiene continuos llamados a la participación de los maestros en el diseño de los diversos componentes del proceso educativo y a la autonomía para manejar la enseñanza, se ha vuelto usual entregarle cada paso perfectamente definido y apabullarlo con tal cantidad de tecnicismos que éste no encuentra posibilidad de desprenderse de ellos..."7.

Así, por ejemplo, la inmensa mayoría de los procedimientos que conducen a la formulación de los currícula así como las características e intencionalidades de los mismos, colocan al maestro en el papel de simple ejecutor: su papel se reduce a interpretar bien los mandatos del currículo para poder ejecutarlo lo más fielmente posible. Diseñar currículos "a prueba de maestros", pareciera que fuera el lema de los diseñadores de los currícula.

No es de extrañarse, entonces, que el maestro en ejercicio desarrolle una actitud muy particular con relación a su campo de trabajo. Actitud que caracterizamos como de "dependencia" y que se ve reforzada por la gran mayoría de acciones y estrategias que instituciones públicas y privadas suelen emprender. Actitud que en alguna manera explica

\footnotetext{
${ }^{6}$ L. Zubieta y O. L. González. EL MAESTRO Y LA CALIDAD DE LA EDUCACIÓN. Revista Educación y Cultura No. 8. FECODE, Santa Fe de Bogotá, julio de 1986.

${ }^{7}$ P. Pinilla, Op. cit., pág. 79.
} 
por qué se ve como natural la necesidad de programas continuados de capacitación: no sólo por parte de las autoridades educativas sino por los mismos maestros (en muchas ocasiones forman parte de sus pliegos de reivindicaciones laborales).

Tal actitud de dependencia es reforzada a su vez por la inmensa mayoría de acciones de capacitación que se suelen llevar a cabo: Acciones de capacitación que se desarrollan a través de cursos que han venido haciendo énfasis, bien sea en que el maestro tenga un mejor dominio de los saberes que enseña (que corrija sus errores y que se ponga al día en el desarrollo de esos saberes) o en que aumente su repertorio de estrategias o técnicas de tipo metodológico para que cumpla con más eficiencia su papel de transmisor del conocimiento. A este respecto, resulta interesante el informe intitulado "L'enseignant aujourd'hui, fonctions, status, politiques", publicado por la OCDE (París), 1990.

El carácter de dependencia desarrollado por el maestro explica en cierto modo la actitud refractaria que suele señalarse como uno de los mayores obstáculos a los esfuerzos de "capacitación" o "actualización" de los docentes en ejercicio.

La actitud de dependencia del maestro está relacionada con su pobre auto imagen y con la forma de asumir su labor cotidiana: no se siente dueño de su quehacer ni con posibilidades de proponer alternativas ni de emprender acciones autónomas en búsqueda de soluciones a los problemas por él detectados, es decir, no se asume como un Profesional.

\section{EL DOCENTE COMO PROFESIONAL}

Asumir las ciencias como la actividad de un grupo social determinado, en contraste a la visión generalizada de ciencia como el conjunto de productos (teorías, procedimientos, aparatos, etc.) de esa actividad, conlleva una nueva concepción sobre el maestro y su quehacer, la cual le posibilita asumir un papel de protagonismo en el avance de su campo de acción. En otras palabras, le posibilita asumirse como un "Profesional de la Enseñanza". Sin embargo, la profesionalización del maestro de Física solo es posible en cuanto se construya una Comunidad Académica de Docentes de la Física, del mismo modo que para referirse a los Profesionales de la Física es necesario hacerlo teniendo como referente la Comunidad de Físicos. Es así como, el Plan de Desarrollo Económico y Social 1990-1994 resalta la necesidad de la formación de una "comunidad científica en educación... que profundice en el entorno social de los procesos educativos y en la relación de educación y desarrollo, para superar la deficiente calidad de la educación”.

El docente es un Profesional cuando su práctica está dirigida por un conjunto sistemático de concepciones, cuando su labor está subordinada a atender las necesidades de todos los sujetos involucrados en el proceso educativo y cuando está en capacidad de organizar y dirigir autónomamente sus prácticas educativas e incidir en la transformación del contexto en el cual se desarrollan. Para que el maestro sea un profesional debe tener una actitud comprometida con el papel que su profesión puede jugar en la construcción de un proyecto cultural que sea la expresión de los intereses de amplios sectores de la sociedad. No basta que sea el gestor de teorías y métodos para orientar su práctica sino que debe incidir en la definición de políticas generales de dirección de la educación. Desde esta perspectiva la dicotomía que usualmente se plantea entre los maestros como los simples usuarios de los resultados de la investigación 
educativa y los investigadores como productores del conocimiento pedagógico, pierde todo sentido. ${ }^{8}$

El reconocimiento de la complejidad de la labor del maestro y de la especificidad de su campo de acción ha conducido, paulatinamente, a reconocer que la calidad de la enseñanza de las ciencias solo se podrá mejorar en la medida en que los profesores comiencen a sentir-se y a actuar como miembros de una comunidad académica, es decir, como Profesionales. El alto porcentaje de los editoriales de la American Journal of Physics en los últimos años dedicados a aspectos relacionados con esta problemática se constituye en una manifestación de este reconocimiento. ${ }^{9}$

\section{SOBRE EL CARÁCTER DE LA PRÁCTICA DEL MAESTRO}

La actividad del maestro es una práctica cultural a través de la cual se actúa en el terreno de la cultura, cuya finalidad consiste en transformar a los sujetos involucrados en los sentidos que ellos lo consideren conveniente para contribuir en la construcción de un proyecto cultural basado en el consenso y no en la enajenación. Asumir que la educación es una práctica cultural, por lo tanto social, implica que las acciones educativas no pueden ser guiadas por criterios exclusivamente técnicos, sino por unas intencionalidades que están íntimamente ligadas a los procesos históricos de la sociedad. Ante esta interpretación de la educación como práctica guiada por intenciones complejas, que a veces son competitivas entre sí y se van modificando a la luz de las circunstancias, debemos comprender la actividad del maestro como una actividad fundamentalmente práctica, históricamente localizada y con consecuencias eminentemente sociales y culturales.

Teniendo en cuenta lo anterior, es claro que las acciones de capacitación de los maestros en ejercicio deben tender a transformar la actitud de dependencia del maestro, lo cual no se puede lograr impartiéndole más contenidos sino dándole elementos que le posibiliten cambiar la concepción de su quehacer, de la disciplina particular que enseña y del conocimiento en general.

\section{LA PROFESIONALIZACION DEL MAESTRO EN EJERCICIO}

Guiados por las anteriores consideraciones, el Programa de Maestría en Docencia de la Física de la Universidad Pedagógica Nacional de Colombia (UPN) cuyo propósito es el de formar investigadores en el campo de la Enseñanza de la Física ha venido desarrollando, además, diferentes estrategias para contribuir en la formación de dicha Comunidad Académica: programas de formación de docentes en ejercicio, actividades y eventos.

\footnotetext{
${ }^{8}$ M. M. Ayala et al. PROPUESTA DE PROGRAMA DE ESTUDIOS CIENT1FICO EN EDUCACIÓN. Programa Nacional de Ciencia y Tecnología de la Educación: Documentos para la Elaboración del Plan. Preimpresos, COLCIENCIAS, Santa Fe de Bogotá, D.C., diciembre de 1991.

${ }^{9}$ Véase por ejemplo: P. B. James. EDITORIAL: A CRISIS IN ATTITUDE. Am. J. Phys. 51(8) Aug.1983.
} 


\section{La formación de maestros en ejercicio}

Para desarrollar la esta estrategia se ha propuesto un programa de especialización en docencia de las ciencias para el nivel básico secundario. El propósito central de este programa de especialización es el de dar elementos que permitan al maestro en ejercicio tener criterios para orientar su práctica. Para tal efecto se busca formar una imagen de ciencia como actividad social (en contraste con un conjunto de teorías ya acabadas), lo cual conlleva a desarrollar una concepción sobre el conocimiento como actividad social. Asumir así el conocimiento permite ver en la enseñanza de una ciencia una actividad eminentemente cultural, y, asumir la ciencia de una manera crítica. Para generar las condiciones de tal transformación en la imagen de las ciencias se plantean tres componentes. Una, de Historia y Epistemología de las ciencias, en la cual se examinan las condiciones que hicieron posible el surgimiento y dieron forma al desarrollo del conocimiento científico. Otra, de Ciencias, con la que se busca generar un espacio para que los maestros se enfrenten al problema de la construcción de los fenómenos y a la construcción de explicaciones. Y la última, la Pedagógica, se concibe como un espacio para la reflexión sobre la práctica de la enseñanza de las ciencias, así como para la construcción de propuestas alternativas teniendo como referencia el desarrollo de las otras dos componentes.

\section{Conformación de una comunidad académica de maestros}

La investigación en cualquier campo del saber no es un problema individual; requiere la construcción de espacios y grupos que lo validen. Para el caso de la enseñanza de las ciencias, campo que apenas se está esbozando, esta consideración resulta fundamental. Se requiere la conformación de una comunidad que reconozca como problemática común la enseñanza de la Física y que se preocupe por buscar elementos teóricos que fundamenten este trabajo. Se requiere la conformación de una comunidad que mediante el examen crítico de sus trabajos conformen y modifiquen sus criterios de validez y los criterios de reconocimiento a sus miembros más prestantes.

A este respecto J. Charum ${ }^{10}$ aporta elementos muy importantes. Es en el laboratorio dice Charum - el lugar en donde se producen los hechos científicos. Todo hecho científico es el resultado de un proceso que tiene como punto inicial la selección y formulación de un problema. Un hecho científico no se reduce a los resultados de un "saber hacer" sustentado en conocimientos adquiridos y ya probados exitosamente en la relación de transformación de la naturaleza. Mas bien demanda la competencia de quienes participan colectivamente en el proceso, de quienes merced a esta competencia, tienen posibilidad de movilizar y articular los conocimientos poseídos, de participar con otros especialistas de otras disciplinas para encarar situaciones nuevas, ejerciendo el control crítico que permite considerar las posibilidades de realizar lo previsto, de redefinir eventualmente el camino para lograrlo, o, incluso cambiar la meta propuesta si la consideración de las circunstancias así lo exigen.

Todo hecho científico — prosigue Charum - necesita de un espacio público calificado donde construir su legitimidad. Un hecho científico para ser tal debe conseguir una aceptación social, no sólo por parte de quienes participaron en su elaboración sino también por parte de aquellos que formal o informalmente participaron en la

$10 \mathrm{~J}$. Charum. ESTRUCTURA CIENTífICA Y ENTORNO SOCIAL. (Anexo 1). Estructura Científica Desarrollo Tecnológico y Entorno Social. Ed. Departamento Nacional de Planeación: Misión de Ciencia y Tecnología. Santa Fe de Bogotá D.C., 1990. 
determinación del problema. Una comunidad académica, entonces, está integrada por grupos y personas que activamente se ocupan de una problemática particular, para conformarla es necesario establecer mecanismos de interacción entre estos grupos. Estos mecanismos de interacción pueden ser: Eventos formales que propicien el encuentro entre los grupos para compartir y confrontar sus logros (Simposios, Encuentros, Congresos), creación de una infraestructura que permita el intercambio continuado aunque no tan formalizado entre los miembros de la comunidad (Bancos de datos, Correo electrónico, etc.), Órganos institucionales para guardar y difundir las memorias del desarrollo del respectivo campo (Revistas especializadas, Revistas de difusión, Academias, Museos...).

Para contribuir a la formación de una comunidad ${ }^{11}$ académica alrededor de la enseñanza de la Física, el programa de Magíster en Docencia de la Física de la UPN ha venido promoviendo actividades tales como:

\section{Los lunes de la enseñanza de la Física}

El grupo "FÍSICA Y CULTURA" ha venido promoviendo 'LOS LUNES DE LA Enseñanza DE LA FÍSICA", actividad que se inició el primer semestre de 1991. Se enfatiza en la participación del sistema educativo que han venido desarrollando investigación y adelantando ensayos y experiencias de innovación, lo cual nos ha permitido iniciar la elaboración de un directorio.

Los “LUNES DE LA ENSEÑANZA DE LA FÍSICA” se orientan a:

- Contribuir a la formación de una cultura académica y científica acorde con las necesidades de nuestro entorno social.

- Promover la formación de una comunidad de pedagogos en ciencias.

- Crear un espacio de comunicación e intercambio de experiencias pedagógicas en la enseñanza de la física y de las ciencias en general

- Difundir las investigaciones que vienen realizando instituciones, grupos de trabajo y personas, sobre la enseñanza de las ciencias.

- Propiciar la reflexión y el debate sobre los problemas tanto teóricos como prácticos de la enseñanza de las ciencias y de la Física en particular.

Esta actividad se ha venido desarrollando mediante ciclos semestrales que incluyen paneles, presentación de experiencias y de propuestas, conferencias, etc. Ha contado con la participación y la asistencia de investigadores, grupos de trabajo y profesores del campo de la enseñanza de las ciencias y de disciplinas afines de diversas instituciones públicas y privadas de los diferentes niveles del sistema educativo.

\footnotetext{
${ }^{11}$ No se trata de una comunidad de "Investigadores", tampoco de "maestros", sino que incluye a todos aquellos que se ocupan de la problemática particular: la Enseñanza de la Física.
} 


\section{Sobre los coloquios}

Resulta interesante notar el poco énfasis que se concede a la realización de congresos de maestros alrededor de las diferentes temáticas propias de su labor profesional, por ser esto un reflejo de la carencia de su profesionalización. Si bien son numerosos los eventos que tanto las universidades como entidades estatales suelen organizar para congregar a los maestros, todos estos eventos se realizan con el ánimo de ejercer una acción sobre ellos, en consecuencia no se les da el carácter de Congreso o similar que suele dársele a eventos realizados por otros profesionales. Sin embargo, últimamente, debido a la consolidación de diferentes grupos de investigación en el campo de la enseñanza de las ciencias, se han comenzado a abrir espacios que posibilitan este tipo de intercambios.

\section{$\underline{\text { Sentido de las redes }}$}

En estos últimos años ha cobrado gran importancia la constitución de "redes de investigadores". La constitución de las redes se plantea como una estrategia para suplir la necesidad de intercambio de trabajos necesario para impulsar la producción de conocimientos, pero es importante tener en cuenta que las redes no pueden suplir la carencia de comunidades académicas. Las redes, así como las bibliotecas, los bancos de datos y otros recursos, son medios que adquieren sentido solo en la medida que contribuyan al fortalecimiento de una comunidad académica. 